\title{
Cryospheric science The power of glacial microbes
}

Organic carbon fluxes from glaciers are a key control on biogeochemical cycles in polar regions. Two analyses of carbon cycling in glaciers show the importance of glacier-surface microbial communities in setting these inputs.

\section{Elizabeth B. Kujawinski}

Glaciers were once considered inhospitable environments for life. However, over the past 15-20 years, vibrant and diverse microbial communities have been documented in all areas of the ice ${ }^{1}$. These communities are generally found in association with liquid water, either between the base of the glacier and the bedrock, or in pores that range in scale from micrometre-scale structures within the ice lattice to larger holes and streams atop the ice itself. However, the balance between the amount of fixed carbon produced by these microbes and that consumed has remained unclear. Writing in Nature Geoscience, Smith et $a l^{2}$. and Musilova et $a l^{3}$. show that the microbial communities fix more carbon than expected and thus play important roles in the glacial carbon cycle in Antarctica and the Greenland Ice Sheet, respectively.

Fixed carbon on glaciers can be approximated by the amount of dissolved organic matter (DOM), but its sources have proven complicated to pinpoint. In particular, the relative contribution of microbes to the consumption of externally-sourced organic matter on the one hand and to organic matter production via photosynthesis on the other hand is unclear. Microbes have been implicated as the primary source of DOM ${ }^{4,5}$, but that is potentially at odds with the radiocarbon values of this organic material which suggest that the carbon comes from an ancient source ${ }^{6}$. The age of the carbon could be explained if the organic matter is a relict from past microbial processes, or microbial breakdown and recycling of ancient carbon. Alternatively, combustion-derived aerosols that have been deposited on the ice carry an ancient radiocarbon signature, and may be a key part of carbon on glacial surfaces in the Northern Hemisphere?

During the height of the melt-season, the organic carbon and nutrients are carried from the glaciers to the sea by glacier run-off and can feed and stimulate marine communities. As such, a clearer understanding of the way in which glacial microbial communities affect carbon fluxes will elucidate the links between glacial and marine biogeochemical cycles.

Smith et al. ${ }^{2}$ address the balance of microbially-mediated carbon fluxes in a supraglacial stream system in Antarctica with an elegant combination of incubation experiments, carbon isotope analyses, and spectroscopy. First, they used spectroscopy to confirm that dissolved organic matter in Antarctic streams was likely derived from microbes. Second, they showed that total microbial carbon production exceeded carbon demand by approximately four times, leaving the excess to be exported to downstream environments. Third, they isotopically labeled organic carbon to monitor carbon uptake by glacial microbes and observed that dissolved organic matter is rapidly utilized by supraglacial microbes. A high fraction of carbon export is consistent with previous reports of microbial carbon delivery to Alaskan and alpine watersheds with high glacial cover ${ }^{6,8}$. This molecular-level demonstration of the power of glacial microbes to 
assimilate and recycle existing microbial organic matter provides experimental confirmation of inferences made by prior studies.

Musilova et $\mathrm{al}^{3}$. approach the question of microbial carbon cycling somewhat differently; they explore microbial communities in small, water-filled holes in the supraglacial system of the Greenland Ice Sheet. Using complementary methods to Smith et $\mathrm{al}^{2}$., they observed overall net organic carbon production, resulting from higher rates of production via photosynthesis than consumption via respiration in all supraglacial habitats during one melt season (May to August). The finding of a net supraglacial carbon surplus in Greenland is thus consistent with the conclusion Smith et al ${ }^{2}$. draw based on their observations in Antarctica.

Moreover, in Greenland, the concentrations of bulk dissolved organic matter as well as compounds that are easier to break down biologically, such as amino acids and carbohydrates, varied over time and habitat in supraglacial dissolved organic matter. This variability underscores the dynamic nature of microbial production and consumption of growth substrates in glacial environments. Despite the northern hemisphere setting, the only supraglacial traces of externally-produced organic matter were found in waters containing significant windblown dust.

Both papers describe an active microbial community - at opposite poles - that has a substantial impact on carbon cycling on the surface of glaciers. This deflates the notion that glacier surfaces are poor hosts for microbial metabolism. Instead, photosynthetic microbes not only support extensive microbial communities that feed off the carbon they produce, but they also generate enough carbon to export reactive dissolved organic matter to downstream environments. In the northern hemisphere, some of these latter non-photosynthetic microbial communities may additionally degrade material from atmospheric deposition; however, Smith et $a l^{2}$. find no evidence of this metabolism in the more remote regions of Antarctica.

Neither study measured radiocarbon values of supraglacial DOM so it is still unclear why the carbon was previously found to be so old. However, evidence is emerging of unexpected light-driven metabolisms in glacial systems, such as carbon monoxide oxidation ${ }^{9}$. This may introduce older sources of inorganic and organic carbon into the pool of actively cycled organic material on the surface of glaciers. Future investigations that can link metabolic pathways with carbon isotopic analyses are required to establish the relative flux and radiocarbon age of carbon through these pathways.

Regardless of the mechanism, Smith et al. ${ }^{2}$ and Musilova et al. ${ }^{3}$ have established that microbial carbon cycling on glacier surfaces cannot be ignored. Microbial activity contributes labile dissolved organic matter to supraglacial and downstream environments where it is combined with organic matter from within or below the glacier. Of course, these organic matter pools are also likely derived from microbes ${ }^{4,5}$, which underscores that these microscopic organisms are the heart of the carbon cycle of glaciers. The exciting next step will be the exploration of the molecular and metabolic details of the rates and extents of microbial processing in these remote habitats.

Elizabeth Kujawinski is at the Woods Hole Oceanographic Institution, 360 Woods Hole Road, Woods Hole, Massachusetts, 02543-1050 U.S.A. email: ekujawinski@whoi.edu 
References

1. Boetius, A., et al., Nature Rev Microbiol., 13, 677-690. (2015)

DOI:10.1038/nrmicro3522

2. Smith, H.J., et al., Nature Geosci., 10, ppp-ppp. (2017) DOI:10.1038/ngeo2925

3. Musilova, M., et al., Nature Geoscience, 10, ppp-ppp. (2017) DOI: 10.1038/ngeo2920

4. Bhatia, M.P. et al., Geochim. et Cosmochim. Acta, 74, 3768-3784. (2010)

DOI:10.1016/j.gca.2010.03.035

5. Pautler, B.G. et al., Env. Sci. Technol., 46, 3753-3761. (2012) DOI:10.1021/es203942y

6. Hood, E., et al., Nature, 462, 1044-1047 (2009) DOI:10.1038/nature08580

7. Stubbins, A., et al., Nature Geosci., 5, 198-201. (2012) DOI:10.1038/ngeo1403

8. Singer, G.A., et al., Nature Geosci., 5, 710-714. (2012) DOI:10.1038/ngeo1581

9. Franzetti, A., et al., ISME J. 10, 2984-2988. (2016) DOI:10.1038/ismej.2016.72

Figure 